\title{
BMJ Open Day of the week to tweet: a randomised controlled trial
}

\author{
Mahesh Jayaram, ${ }^{\oplus 1,2}$ Clive E Adams, ${ }^{3}$ Johannes S Friedel, ${ }^{4}$ Eimear McClenaghan, ${ }^{5}$ \\ Alan A Montgomery, ${ }^{6}$ Maritta Välimäki, ${ }^{7,8}$ Lena Schmidt, ${ }^{4}$ Jun Xia, ${ }^{9,10}$ Sai Zhao ${ }^{11}$
}

To cite: Jayaram M, Adams CE, Friedel JS, et al. Day of the week to tweet: a randomised controlled trial. BMJ Open 2019;9:e025380. doi:10.1136/ bmjopen-2018-025380

- Prepublication history for this paper is available online. To view these files, please visit the journal online (http://dx.doi org/10.1136/bmjopen-2018025380).

Received 25 January 2019 Revised 7 February 2019 Accepted 12 February 2019

\section{Check for updates}

(c) Author(s) (or their employer(s)) 2019. Re-use permitted under CC BY-NC. No commercial re-use. See rights and permissions. Published by BMJ.

For numbered affiliations see end of article.

\section{Correspondence to} Dr Mahesh Jayaram; mahesh.jayaram@unimelb. edu.au

\section{ABSTRACT}

Objective To assess the effects of using health social media on different days of the working week on web activity.

Design Individually randomised controlled parallel group superiority trial.

Setting Twitter and Weibo.

Participants 194 Cochrane Schizophrenia Group full reviews with an abstract and plain language summary web page. There were no human participants.

Interventions Three randomly ordered slightly different messages (maximum of 140 characters), each containing a short URL to the freely accessible summary page, were sent on specific times on a single day. Each of these messages sent on Tuesday, Wednesday, Thursday and Friday was compared with the one sent on Monday. Outcome The primary outcome was visits to the relevant Cochrane summary web page at 1 week. Secondary outcomes were other metrics of web activity at 1 week. Results There was no evidence that disseminating microblogs on different days of the working week resulted in any differences in target website activity as measured by Google Analytics ( $n=194$, all page views, adjusted ratios of geometric means 0.86 (95\% $\mathrm{Cl} 0.63$ to 1.18), 0.88 (95\% Cl 0.64 to 1.21), 0.88 (95\% Cl 0.65 to 1.21), 0.91 (95\% Cl 0.66 to 1.24) for Tuesday-Friday, respectively, overall $\mathrm{p}=0.89$ ). There were consistent findings for all outcomes. However, activity on the review site substantially increased compared with weeks preceding the intervention.

Conclusion There are no clear differences in the effect when 1 weekday is compared with another, but our study suggests that using microblogging social media such as Twitter and Weibo do increase information-seeking behaviour on health. Tweet any day but do Tweet.

\section{INTRODUCTION}

The Cochrane Collaboration is a network of researchers, healthcare professionals, patients, carers and people interested in healthcare who largely work for free with altruistic motives to produce systematic reviews of care. ${ }^{1}$ Cochrane systematic reviews are available in the Cochrane Library (http:/ / www.cochranelibrary.com/) and can be freely accessed in 101 countries, available to residents of a further 14 countries via subscription paid by respective governments or other organisations. It is also available for purchase
Strengths and limitations of this study

Sample size was small limiting the power to highlight real differences.

- Free-to-use software limited functionality and sophisticated software may highlight more effects.

- Tweeted links to large academic reviews on one area of healthcare to a relatively small 'followings' could limit impact.

- Different techniques of microblogging, linking to less 'weighty' documents and a large, broad set of followers may result in more impact.

at a cost or as open access if authors have paid the article processing charge. ${ }^{2}$ Each full review has a summary and abstract web page accessible to anyone with internet access ${ }^{3}$ (http://www.cochrane.org/). Cochrane Schizophrenia Group (CSzG) sits within this wider Collaboration.

Twitter is a popular ${ }^{4}$ free to use microblogging social media platform, which, at the time of the trial, allowed users to send a 140 character message called a tweet to lists of followers. A tweet may contain a hashtag (\#), used as a means of searching by topic, and/or a handle (@), which denotes another Twitter user account. Although Twitter (blocked in China since 2009), and some other platforms are not available in China, the usage of social media is exceptionally high with $91 \%$ of Chinese residents using some form of social media compared with about $67 \%$ of those in the USA. ${ }^{5}$ The Twitter-like systems of Weibo and Wechat are popular in China. ${ }^{67}$

In 2011, CSzG began to use Twitter. @ CochraneSzGroup following remains small but at the time of the study was over 1300 . Our randomised trial (tweeting links vs not tweeting links of similar paired reviews ${ }^{8}$ showed that social media dissemination of reviews nearly tripled the unique page visits to each review and nearly increased threefold the time people spent visiting the Cochrane summaries page when they followed the link via a tweet. ${ }^{9}$ We subsequently identified a 
Table 1 Past analyses of differences in activity across weekdays

\begin{tabular}{|c|c|c|c|c|}
\hline Source & Method & Measuring & Data set & Conclusion \\
\hline How to get more clicks on Twitter ${ }^{11}$ & Case series & $\mathrm{CTR}^{*}$ & 200000 tweets & Weekends \\
\hline Time is on your side ${ }^{15}$ & Case series & CTR & Unknown tweets & Weekdays \\
\hline $\begin{array}{l}\text { Twitter marketing - what results should } \\
\text { we expect? }{ }^{13}\end{array}$ & Survey & CTR & 20000 tweets & Weekends \\
\hline $\begin{array}{l}\text { Cascading behaviour in large blog } \\
\text { graphs patterns and a model }\end{array}$ & Case series & CTR & $21.3 \mathrm{~m}$ blog posts & Weekdays \\
\hline $\begin{array}{l}\text { The Biggest Social Media Science } \\
\text { Study: What } 4.8 \text { million Tweets Say } \\
\text { About the Best Time to Tweet }{ }^{18}\end{array}$ & Survey & $\begin{array}{l}\text { CTR, time to tweet, } \\
\text { engagement with tweet }\end{array}$ & $4.8 \mathrm{~m}$ tweets & Variable results \\
\hline
\end{tabular}

${ }^{*}$ CTR: Ratio of users who click on a specific link to the number of total users who view a page.

CTR, click-through rate.

variety of case reports, surveys and case series' conclusions on when to microblog (table 1). Each study stresses the importance of appreciating weekly behaviour patterns when tweeting and how to increase traffic as more tweets mean more visibility. In contrast to other social media such as Facebook, Twitter activity dies down a bit during weekends. ${ }^{10}$ For studies that compare 1 day of the week against another, conclusions are conflicting as are comparisons between weekday and weekend tweeting. Four support microblogging (tweeting) at the weekend ${ }^{11-14}$ and three during the 'working' week. ${ }^{15-17}$ One large survey of 4.8 million tweets reported that early morning tweets received most clicks while late evening tweets had more retweets. ${ }^{18}$ There is peak tweeting activity between the hours of 11:00 and 13:00. The best days and times to microblog in terms of site traffic are likely to differ according to the target audience. For example, tweets for shoppers may be best at the end of the week when most are shopping, and tweets of work-related information may be better during the working week.

Given the above findings, we considered examined our own@CochraneSZGroup account by analysing data from Twitter Analytics ${ }^{19}$ for the period 1 June 2015 to 25 October 2015 (figure 1). We found the @ CochraneSZGroup engagement rate to be higher during the working week than at the weekend, with the greatest activity on Thursday and Friday.

\section{Objective}

To evaluate whether structured microblogging of treatment evidence on any particular day of the working week (Monday-Friday) increases the 'all page views' metric recorded by Google Analytics. 'All page views' is the total number of visits to the target page. This generic outcome is routinely collected by the Google Analytics package and has been used in past work.

\section{METHODS AND ANALYSIS Study design}

Prospective two-arm, parallel, open randomised controlled trial with an equal allocation. Protocol with full details of the study design has been published elsewhere. ${ }^{20}$

\section{Patient and public involvement}

Cochrane library and the Cochrane summaries pages are widely accessed by the general population, patients and carers as well as those within the healthcare industry. The research question arose from an interest in ensuring that the information within these webpages is disseminated as widely as possible and that this is done in a manner that is innovative using social media. This process of course has to have an evidence base and hence the idea of conducting a trial to evaluate whether disseminating evidence in this manner works or not. The participants in this trial were not patients, but rather systematic reviews themselves.

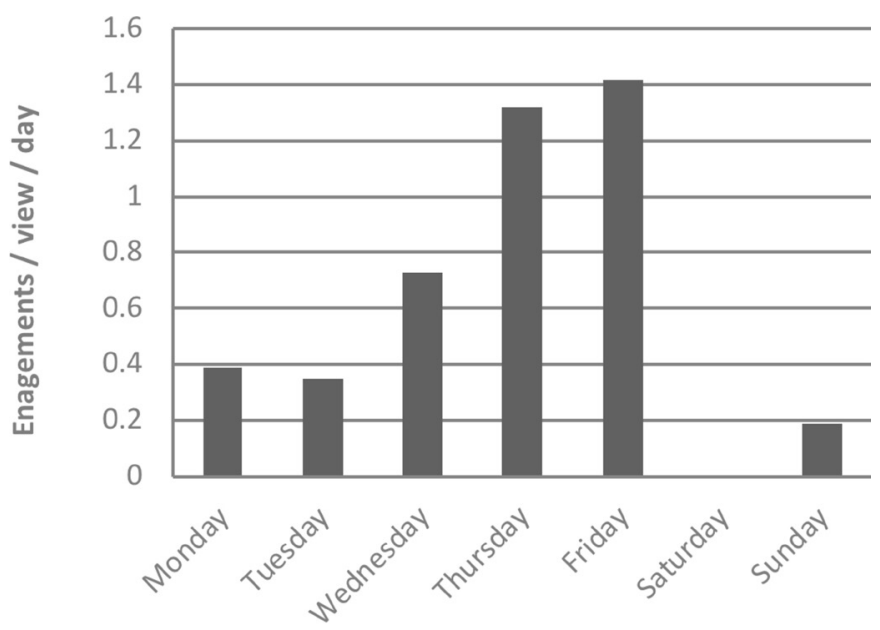

Figure 1 Average engagement* rate (1 June 2015 to 25 October 2015). *Engagement rate is a broad measure of Twitter activity encompassing CTR and Twitter interactions. 
Hence, patients were not involved in the study. There was no recruitment as such as all the systematic reviews were already published in the Cochrane library and hence the dissemination of results to patients also does not apply. Again for the above reason, the burden of intervention assessment by patients also does not apply. Patients and public were not involved in this trial.

\section{Participants}

Participants in this study were published full-text $\mathrm{CSzG}$ systematic reviews (date reviews published were between 2000 and 2015) in The Cochrane Library with Plain Language Summary (PLS) which is freely available at www.summaries.cochrane.org. We excluded published protocols for $\mathrm{CSzG}$ reviews, any $\mathrm{CSzG}$ review not relevant to schizophrenia, unpublished and withdrawn CSzG reviews.

This study was conducted entirely online. The participant PLSs were all available on the Cochrane Library website, the intervention was delivered via two social media platforms (Twitter and Weibo) and outcome data were collected using Google Analytics.

This study did not involve any living participants and used information that is available in the public domain. Participants are systematic reviews rather than people and routine web activity data were extracted and recorded through Google Analytics. No ethical approval was required. ${ }^{21} 22$

\section{Randomisation}

All participating CSzG systematic reviews were identified prior to randomisation. We defined reviews by tertiles of baseline access activity during the 52-week period, 1 January 2015 to 31 December 2015: high (527 to 4023 hits, $\mathrm{n}=64$ ), medium (232 to 523 hits, $\mathrm{n}=65$ ) or low (45 to 230 hits, $\mathrm{n}=65$ ). Reviews were then assigned a unique code, which, along with baseline access activity stratum was supplied to one of the authors (AAM) who generated the random number sequence using a computer and performed randomisation. Stratifying by baseline activity, and using a computer-generated random number sequence, reviews were first allocated to day of the week Monday through Friday then into week number (1-39), and finally, the order in which the three microblogs (title, extract and question) would be released. Tweets had been prepared for all reviews. Allocations were then supplied by AAM to EM and SZ who, independent of other investigators, scheduled tweets for Twitter and Weibo/WeChat. We created two new usernames @CochraneSzFIN (78 followers) and @CochraneSzAuz (74 followers) to disseminate the same tweets in the Finnish language and to Australia in English.

\section{Procedures}

For the 10 weeks preceding randomisation, 20 microblogs about research relevant to schizophrenia from Mental $^{23}$ were Tweeted in three tweet bundles. Tweets were split evenly to each day of the week. This ensured @
Box 1 Example of the three tweets relating to same review

Tweet 1: \#Clozapine combined with different \#antipsychotic \#drugs for \#treatment resistant \#schizophrenia http://ow.ly/yaKAU

Tweet 2: How effective is \#clozapine in addition to another \#antipsychotics at treating 'hard to treat' \#schizophrenia? http://ow.ly/yaKAU Tweet 3: Not clear if combining \#clozapine with other \#antipsychotics is effective for \#treatment resistant \#schizophrenia http://ow.ly/yaKAU

CochraneSZGroup was active before the start of the trial as our activity had declined across 2015.

Between 26 January 2016 and 20 October 2016, one review in a day was tweeted in English, Finnish and Mandarin, by the $\mathrm{CSzG}$, three times on the allocated day at 10:30, 13:00 and 15:00 hours local time. These timings are suggested by the SocialBro web tool as when people are likely to be most receptive. ${ }^{24}$ There is some evidence that multiple postings, 3-4 times a day, of the same or similar tweet can be useful for an international following. ${ }^{25}$ Each of the three tweets had different text:

- The review title as it appears in www.summaries. cochrane.org, and a shortened URL to the summaries web page.

- A pertinent extract from the results or discussion sections of the abstract, and a shortened URL to the summaries web page.

- An intriguing question or pithy statement directly related to the evidence presented in the abstract and a shortened URL to the summaries web page (see box 1).

The study was designed to compare the effects of sending this package of tweets on different days of the week, rather than the specific content of the different types of accompanying text, which were formulated in order to appeal to the wide range of followers of the CSzG Twitter page and searchers. To assist the logistics of sending out tweets, we used Hootsuite, a social media management system. ${ }^{26}$ This free package allows formulation and scheduling of Tweets and is now available in China and integrates with Weibo. ${ }^{27}$

\section{Outcomes}

The primary outcome was the total number of visits to the relevant Cochrane summary page in the 7 days following the intervention (including the day of tweeting) as reported on Google Analytics. For the primary outcome, repeated views of the same page during a single user session are counted in the total (unique page views are a secondary outcome). The standard free account in Google Analytics produces various reports in real time and data about whom, when and where someone has visited a site as well as how they 'arrived' there. ${ }^{9}$ In this way, we monitored all traffic to the Cochrane summary page and traffic directly from Twitter. The average halflife of a tweet (with a web link), defined as 'the amount of time at which this link will receive half of the clicks 


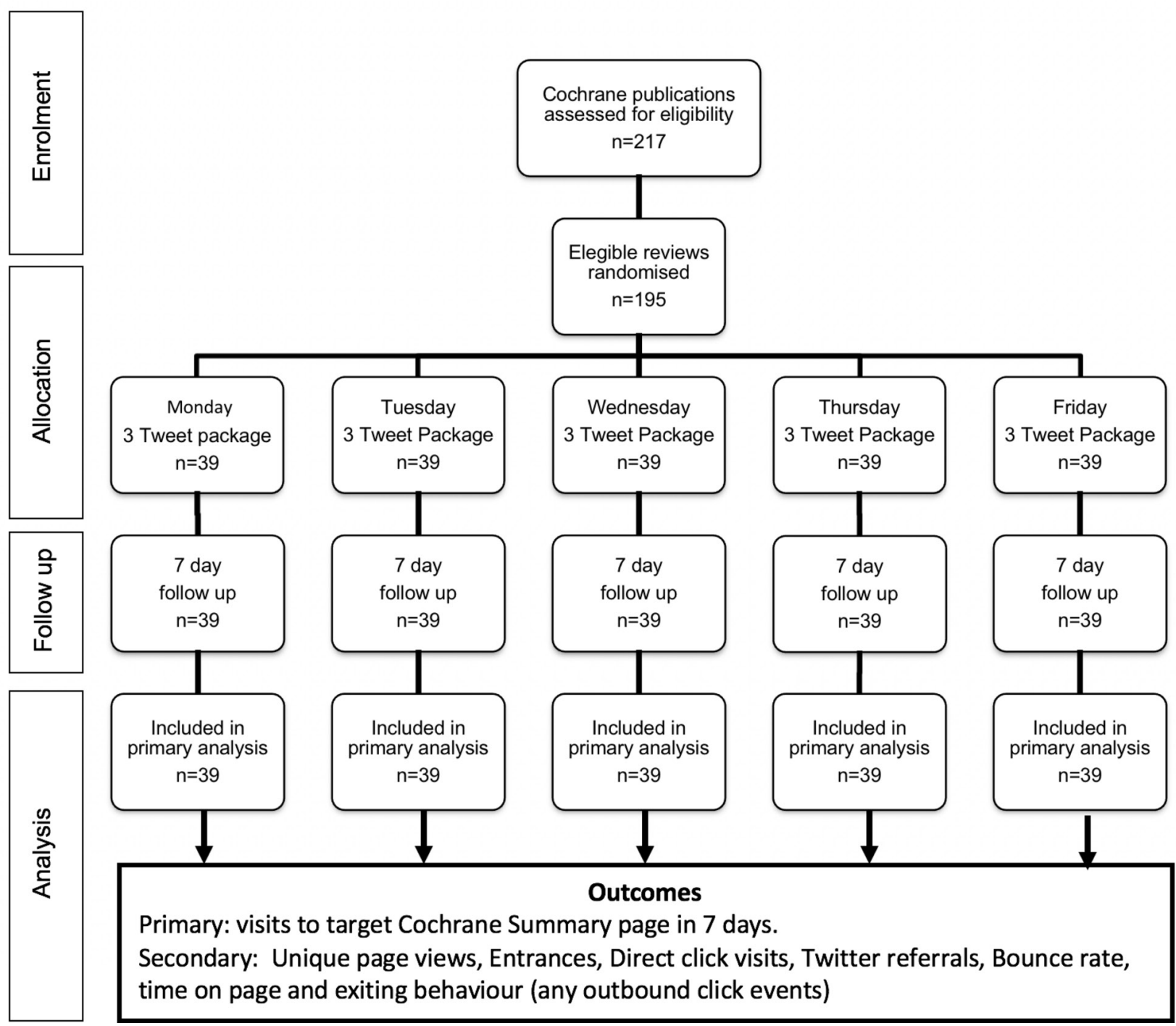

Figure 2 Flow diagram.

it will ever receive after it's reached its peak', has been estimated as 2.8 hours. ${ }^{28}$ However, to capture any possible cascade effect of tweeting, we extended the monitoring period to 7 days. ${ }^{29}$ The secondary outcomes are listed in figure 2 and provide other measures of incoming activity and exiting behaviour. For a glossary of Google Analytics terms, please see ref 20. After the final follow-up period, JSF downloaded data from Google Analytics using Supermetrics (business analytics software) ${ }^{30}$ to produce a spreadsheet for analysis.

\section{Statistical power}

The sample size for this study is fixed by the number of published Cochrane reviews within the jurisdiction of the $\mathrm{CSzG}(\mathrm{n}=194)$. As a multiarm trial (five arms) with no natural control arm for comparison and a log-normal continuous primary outcome, analysis of the primary outcome is by analysis of covariance where the effect size is the ratio of between-arm and residual variances. The detectable (standardised) effect size is calculated using standard sample size software after specifying power, alpha, number of arms and number of observations per arm. This represents the ratio of between-arm and residual variances. In a one-way analysis of variance study, sample sizes of 39, 39, 39, 39 and 39 are obtained from the 5 groups whose means are to be compared. The total sample of 194 achieves $90 \%$ power to detect differences among the means versus the alternative of equal means using an $\mathrm{F}$ test with a 0.05 significance level. The detectable standardised effect size assumes a common SD within a group of 1.00. Therefore, the size of the variation in the means is represented by their SD which is 0.28 . After taking logs of baseline number of visits for each review, the actual common SD within a group is 0.93 . With a standardised detectable effect size of $0.28 \%$ and $90 \%$ power, this equates to a between-group SD of approximately 0.26 or variance $\left(=\mathrm{SD}^{2}\right)$ of approximately 0.07 .

\section{Data analysis}

We compared baseline access activity of the reviews in each of the five trial arms using descriptive statistics. We analysed all outcomes as continuous, with log transformation 
Table 2 Baseline activity (or views) for 12 month period preceding randomisation (January-December 2015)

\begin{tabular}{llllllll}
\hline \multirow{2}{*}{$\begin{array}{l}\text { Randomised } \\
\text { group }\end{array}$} & $\mathbf{N}$ & \multicolumn{2}{l}{ Views } & & & \\
\cline { 2 - 7 } & Mean (SD) & Minimum & 25th centile & Median & 75th centile & Maximum \\
\hline Monday & 39 & $597(750)$ & 89 & 175 & 325 & 662 & 4023 \\
Tuesday & 39 & $594(698)$ & 68 & 203 & 338 & 630 & 3594 \\
Wednesday & 39 & $473(648)$ & 45 & 141 & 292 & 640 & 4010 \\
Thursday & 39 & $582(585)$ & 61 & 205 & 387 & 801 & 620 \\
Friday & 38 & $536(641)$ & 73 & 190 & 268 & 3304 \\
\hline
\end{tabular}

for outcomes that were positively skewed. We present descriptive statistics, and measures of effect from linear regression models using Monday as a nominal reference group. For the primary outcome and most secondary outcomes, this estimate of effect is the ratio of geometric means and 95\% CIs, adjusted for baseline activity. However, as a multiarm study with no pairwise comparisons of particular interest, we derived a single $p$ value for each outcome based on a Wald test that the model coefficients for treatment arm are jointly equal to zero. All data were analysed using Stata V.15 statistical software.

As the allocated interventions were implemented fully as planned, and there were no missing primary outcome data, we did not conduct any sensitivity analyses.

\section{RESULTS}

Following randomisation, 194 reviews were tweeted. Table 2 outlines the baseline review activity for the year before the start of the trial. The minimum and maximum correspond to the least and most viewed reviews by allocated day of the week, respectively. There were no differences between the groups.

For this randomised trial, primary and secondary findings were consistent. Tweeting on any day between Monday and Friday was just as good as any other weekday and no clear advantage over another in terms of ratings of activity as recorded in Google Analytics (table 3). Randomised evidence from our previous trial ${ }^{31}$ suggested an approximately threefold increase in activity in the week after disseminating microblogs with hyperlinks. This study provides before-and-after evidence that this level of increase in activity continues to be realistic.

\section{DISCUSSION}

Results from this study demonstrate that there was no statistically significant difference between tweeting on Monday or any day of the week for our primary outcome of 'all page views'. Equally, there were no clear differences found for any of the secondary outcomes. There are many possibilities why this was so. It is possible that a real effect has failed to be detected in this necessary small trial or the form of the microblogs may not have encouraged differential responses. Perhaps, however, for this type of tweet and Twitter following there is no substantial advantage to microblogging on one working day compared with another.

There was, however, an increase in viewing activity compared with the months prior to the study. This fits with the finding of our previous randomised trial. ${ }^{31}$ In that 2013-2014 study, the control group had an arithmetic mean of 5.28 'all page views' and this increased in the tweet arm to 13.67. After that trial ended, there was a general increase in baseline activity as evidenced by the data we collected for the year prior to this trial (table 2). Despite this increased baseline activity, tweeting in this study seemed to increase activity by a further threefold. There is a suggestion that interest is increasing and that this has not plateaued. The other comparable variable between the two trials is 'time spent on page'. In this study, time on page was considerably greater than in our first trial (geometric mean 123 vs 76 s).

Much investment is made to disseminate evidence of the effects of healthcare but the strategies by which this is done are rarely investigated. Increasingly, more funding bodies are prioritising dissemination strategies as part of evaluating successful grant applications and decision-making tools are available to support this. ${ }^{32} 33$ Medical evidence of enormous impact will disseminate swiftly by every means possible with very little effort beyond the point of disclosure of findings. Maximising impact of most other health evidence does take more effort beyond the publication of the final report. Many choices are available to individuals, institutions and industry and resources limit what can be done but social media is ubiquitous, inexpensive and-as this study continues to suggest-powerful. The first randomised trial in our series illustrated that using a microblog causes nearly a tripling of traffic to a health evidence web page. ${ }^{31}$ This study supports that finding with the non-randomised before and after hit rates but adds randomised evidence that which day of the week dissemination takes place on does not matter that much. This higher quality evidence does not support the impression we gained form initial routine data analysis (table 1 , figure 1 ) and, again, highlights the value of gaining evidence from randomised trials if possible before embarking on policy based solely on routine data. Perhaps with greater numbers and more power, we would have shown a real difference favouring Monday over the other days as this seemed the only day 
Open access

Table 3 Results

\begin{tabular}{|c|c|c|c|c|c|c|c|}
\hline Group & $\mathbf{N}$ & $\begin{array}{l}\text { Arithmetic } \\
\text { mean }\end{array}$ & SD & $\begin{array}{l}\text { Geometric } \\
\text { mean }\end{array}$ & $\begin{array}{l}\text { Adjusted ratio } \\
\text { of geometric } \\
\text { mean }\end{array}$ & $95 \% \mathrm{Cl}$ & P value* \\
\hline \multicolumn{8}{|c|}{ Primary outcome } \\
\hline \multicolumn{8}{|c|}{ All page views } \\
\hline Monday & 39 & 36 & 43 & 22 & - & - & \\
\hline Tuesday & 39 & 26 & 22 & 19 & 0.86 & 0.63 to 1.18 & \\
\hline Wednesday & 39 & 24 & 24 & 17 & 0.88 & 0.64 to 1.21 & \\
\hline Thursday & 39 & 30 & 28 & 19 & 0.88 & 0.65 to 1.21 & \\
\hline Friday & 38 & 29 & 34 & 19 & 0.91 & 0.66 to 1.24 & 0.89 \\
\hline \multicolumn{8}{|c|}{ Secondary outcomes } \\
\hline \multicolumn{8}{|c|}{ Unique page views } \\
\hline Monday & 39 & 33 & 41 & 20 & - & - & \\
\hline Tuesday & 39 & 23 & 19 & 17 & 0.87 & 0.64 to 1.18 & \\
\hline Wednesday & 39 & 21 & 21 & 15 & 0.88 & 0.65 to 1.19 & \\
\hline Thursday & 39 & 27 & 26 & 17 & 0.90 & 0.67 to 1.22 & \\
\hline Friday & 38 & 25 & 29 & 17 & 0.90 & 0.66 to 1.22 & 0.90 \\
\hline \multicolumn{8}{|l|}{ Entrances } \\
\hline Monday & 39 & 29 & 39 & 16 & - & - & \\
\hline Tuesday & 39 & 18 & 15 & 13 & 0.81 & 0.58 to 1.13 & \\
\hline Wednesday & 39 & 17 & 19 & 12 & 0.85 & 0.61 to 1.19 & \\
\hline Thursday & 39 & 22 & 24 & 13 & 0.82 & 0.59 to 1.15 & \\
\hline Friday & 38 & 22 & 28 & 13 & 0.88 & 0.63 to 1.23 & 0.74 \\
\hline \multicolumn{8}{|c|}{ Direct click visits } \\
\hline Monday & 39 & 5 & 5 & 3 & - & - & \\
\hline Tuesday & 39 & 4 & 5 & 3 & 0.98 & 0.69 to 1.40 & \\
\hline Wednesday & 39 & 3 & 3 & 2 & 0.77 & 0.54 to 1.09 & \\
\hline Thursday & 39 & 4 & 4 & 3 & 0.91 & 0.64 to 1.29 & \\
\hline Friday & 38 & 4 & 4 & 3 & 0.87 & 0.61 to 1.24 & 0.58 \\
\hline \multicolumn{8}{|c|}{ Twitter referrals } \\
\hline Monday & 39 & 5 & 9 & 3 & - & - & \\
\hline Tuesday & 39 & 4 & 4 & 3 & 0.81 & 0.52 to 1.26 & \\
\hline Wednesday & 39 & 3 & 3 & 3 & 0.76 & 0.48 to 1.18 & \\
\hline Thursday & 39 & 4 & 3 & 3 & 0.92 & 0.59 to 1.44 & \\
\hline Friday & 38 & 4 & 3 & 3 & 0.90 & 0.57 to 1.41 & 0.75 \\
\hline \multicolumn{8}{|c|}{ Outbound click events } \\
\hline Monday & 39 & 5 & 6 & 2 & - & - & \\
\hline Tuesday & 39 & 5 & 5 & 3 & 1.19 & 0.72 to 1.98 & \\
\hline Wednesday & 39 & 5 & 6 & 3 & 1.20 & 0.72 to 2.00 & \\
\hline Thursday & 39 & 4 & 5 & 2 & 0.93 & 0.56 to 1.54 & \\
\hline Friday & 38 & 4 & 4 & 3 & 1.11 & 0.67 to 1.86 & 0.82 \\
\hline \multicolumn{8}{|c|}{ Time on page (seconds) $\dagger$} \\
\hline Monday & 36 & 197 & 249 & 135 & - & - & \\
\hline Tuesday & 37 & 179 & 265 & 96 & 0.71 & 0.45 to 1.12 & \\
\hline Wednesday & 36 & 200 & 168 & 127 & 0.97 & 0.62 to 1.54 & \\
\hline Thursday & 36 & 190 & 172 & 132 & 0.98 & 0.62 to 1.55 & \\
\hline
\end{tabular}

Continued 
Table 3 Continued

\begin{tabular}{|c|c|c|c|c|c|c|c|}
\hline Group & $\mathbf{N}$ & $\begin{array}{l}\text { Arithmetic } \\
\text { mean }\end{array}$ & SD & $\begin{array}{l}\text { Geometric } \\
\text { mean }\end{array}$ & $\begin{array}{l}\text { Adjusted ratio } \\
\text { of geometric } \\
\text { mean }\end{array}$ & $95 \% \mathrm{Cl}$ & P value* \\
\hline \multirow[t]{2}{*}{ Friday } & 35 & 185 & 168 & 129 & 0.97 & 0.61 to 1.54 & 0.52 \\
\hline & & & & & $\begin{array}{l}\text { Adjusted } \\
\text { difference in } \\
\text { means }\end{array}$ & & \\
\hline
\end{tabular}

Per cent of visits that were single page

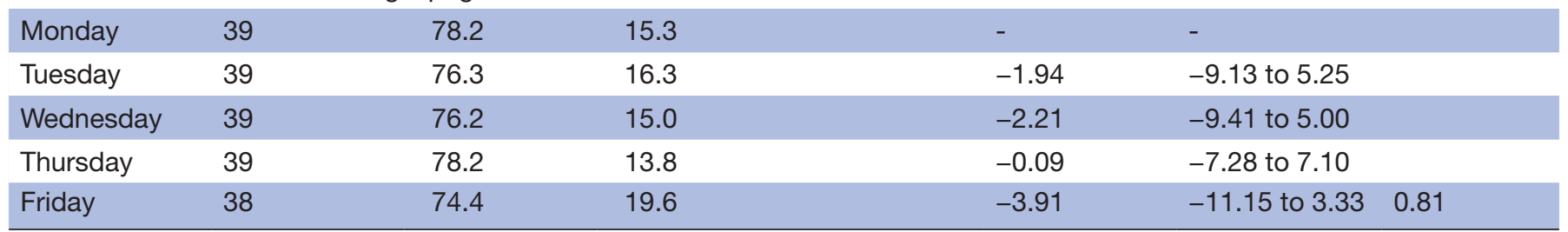

${ }^{*} P$ value based on Wald test that coefficients are jointly equal to zero.

†A few of the reviews had zero visits, hence cannot contribute to this outcome.

to have a little more activity than any of the others. This, however, is conjecture and any difference, for this set of evidence, for this following, would be subtle. Our trial tweeted between the hours of 10: 30 and 15: 00 hours local time and this could be a limitation in the local context where it is possible that more professional social media users have sight of it. However, given the global reach of tweets and access across time zones, we believe the impact of this would be minimal. Perhaps further studies could explore this aspect.

There remain many more questions on which type of social media delivers best impact on health behaviour, and on what content, timing and targeting maximally encourages best evidence to be considered and then implemented. Few techniques or tools have been demonstrated to swiftly influence behaviour of those seeking information on health. Social media are accessible tools and it is possible to evaluate how we use them and then use them in a way that does not waste effort or interest.

\section{CONCLUSIONS}

Use of social media in an area of healthcare that is not particularly high profile, for dissemination of evidence that is highly specialised to a small following continues to have a genuine effect on increasing indicators of interest-but we have no evidence that use of social media on any particular working day of the week matters. This study continues to support the initial finding from the first randomised trial where the use of social media (Twitter/ Weibo) almost immediately nearly tripled activity on the reviews' summary page. For those interested in wider dissemination of evidence, tweet any working day, but do tweet.

\section{Ethics and dissemination}

This study does not involve any living participants and uses information that is available in the public domain.
Participants in this study are systematic reviews rather than people. The http://www.cochrane.org/ and the Cochrane Library websites will be the target outcomes and the routine data will be extracted and recorded through Google Analytics. As a result, no ethical approval is required. ${ }^{21}{ }^{22}$ Results will be disseminated via Twitter, Weibo and other traditional academic means.

\section{Trial organisation}

This study did not have a data monitoring committee or a steering committee.

\section{Author affiliations}

${ }^{1}$ Department of Psychiatry, University of Melbourne, Melbourne, Victoria, Australia

${ }^{2}$ Psychiatry, Melbourne Health, Melbourne, Victoria, Australia

${ }^{3}$ School of Medicine, University of Nottingham, Nottingham, UK

${ }^{4}$ Hochschule Furtwangen University of Applied Sciences, Furtwangen, BadenWürttemberg, Germany

${ }^{5}$ Medical School, University of Aberdeen Institute of Applied Health Sciences, Aberdeen, UK

${ }^{6}$ Nottingham Clinical Trials Unit, University of Nottingham, Nottingham, UK ${ }^{7}$ Department of Nursing Science, University of Turku, Turku, Finland

${ }^{8}$ Department of Nursing, The Hong Kong Polytechnic University, Hong Kong, Hong Kong

${ }^{9}$ Systematic Review Solutions Limited, University of Nottingham, Nottingham, UK

${ }^{10}$ Nottingham Health China, The University of Nottingham, Ningbo, China

${ }^{11}$ Systematic Review Solutions Limited, Ningbo, China

Acknowledgements We are really grateful to Julie Wood, Head of External Affairs and Communications, Cochrane Central Executive and Paolo Rosati Web Operations Manager, Informatics and Knowledge Management Department, Cochrane Central Executive who were supportive of the idea and practical in helping it move forward. Farhad Shokraneh, Information Specialist of the Cochrane Schizophrenia Group is, as always, a rock of support and help in times of need.

Contributors MJ, CEA, AAM, MV and EM designed the protocol and helped draft the manuscript. CEA and EM wrote the tweets and coordinated the UK, Finland and Australia arm of the trial. JSF and LS wrote the computer program to retrieve data from Google Analytics. AAM analysed the data. SZ translated tweets into Chinese and JX coordinated the China/Weibo/Wechat arm of the trial. MV translated tweets into Finnish. MJ and CEA finalised the manuscript, double checked the data and hold all data. MJ has coordinated all revisions and re submissions. MJ will make data available through the University of Melbourne server. All authors provided critical feedback and helped shape the research, analysis and manuscript. All 
authors are accountable for accuracy and integrity of the work. All authors read and approved the final manuscript.

Funding Intramural funding from the University of Nottingham, University of Melbourne and the University of Aberdeen. Economic and Social Research Council Impact Acceleration Account (IAA) funding to support conduct and dissemination of this work (ESRC IAA University of Nottingham).

Competing interests None declared.

Patient consent for publication Not required.

Provenance and peer review Not commissioned; externally peer reviewed.

Data sharing statement The data will be made available via the University of Melbourne repository.

Open access This is an open access article distributed in accordance with the Creative Commons Attribution Non Commercial (CC BY-NC 4.0) license, which permits others to distribute, remix, adapt, build upon this work non-commercially, and license their derivative works on different terms, provided the original work is properly cited, appropriate credit is given, any changes made indicated, and the use is non-commercial. See: http://creativecommons.org/licenses/by-nc/4.0/.

\section{REFERENCES}

1. The Cochrane Collaboration. [Internet]. 2014 [cited 27 Oct 2015]. Available from: http://www.cochrane.org/about-us/evidence-basedhealth-care

2. The Cochrane Library. 2014. http://www.thecochranelibrary.com/ view/0/FreeAccess.html (cited 2015 Oct 27).

3. Mcllwain C, Santesso N, Simi S. Standards for the reporting of Plain Language Summaries in new Cochrane Intervention Reviews (PLEACS). 2015. http://editorial-unit.cochrane.org/sites/editorial-unit. cochrane.org/files/uploads/PLEACS 0.pdf (cited 27 Oct 2015).

4. Twitter. Our mission: To give everyone the power to create and share ideas and information instantly, without barriers. 2015. https://about. twitter.com/company (cited 27 Oct 2015).

5. Go-Globe. Social Media In China-Statistics and Trends [Infographic] [Internet]. 2013. http://www.go-globe.com/blog/social-media-china (cited 2015 Oct 27).

6. Smith C. By the Numbers: 37 Amazing Weibo Statistics. 2015. http:// expandedramblings.com/index.php/weibo-user-statistics (cited 27 Oct 2015).

7. Smith C. By the Numbers: $50+$ WeChat Statistics [Internet]. http:// expandedramblings.com/index.php/wechat-statistics/.

8. Jayaram M, Bodart AY, Sampson S, et al. To tweet or not to tweet about schizophrenia systematic reviews (TweetSz): study protocol for a randomised controlled trial. BMJ Open 2015;5:e007695.

9. Google. Google Analytics. [Internet]. 2015. http://www.google.com/ analytics (cited 2015 Oct 27)

10. Kolowich L. The Best Time to Post on Instagram, Facebook, Twitter, Linkedln, Pinterest, and Google+ [Infographic] [Internet]. https://blog. hubspot.com/marketing/best-times-post-pin-tweet-social-mediainfographic (cited 30 Jan 2018).

11. Danzarella. How to Get More Clicks [Internet]. 2015. http:// danzarrella.com/infographic-how-to-get-more-clicks-on-twitter.html (cited 26 Oct 2015).
12. Alwagait E, Shahzad B. When Are Tweets Better? J Univers Comput Sci 2015;20:1511-21.

13. Sign Up To. Twitter Marketing-What Results Should You Expect? [Internet]. 2015. http://www.signupto.com/news//10/24/twittermarketing-what-results-should-you-expect-infographic (cited 26 Oct 2015).

14. Calculating and Improving Your Twitter CTR [Internet]. https://moz. $\mathrm{com} / \mathrm{blog} / \mathrm{calculating}$-and-improving-your-twitter-clickthroughrate (cited 26 Oct 2015).

15. Mason H. Time Is On Your Side. [Internet]. Bitly. 2015. http://blog. bitly.com/post/22663850994/time-is-on-your-side (cited 27 Oct 2015).

16. Rao A, Li Z, Bhattacharyya P, et al. When-To-Post on Social Networks [Internet]. 2015. http://arxiv.org/pdf/1506.02089v1.pdf (cited 2015 Oct 30).

17. Leskovec J, McGlohon M, Faloutsos C, et al. Cascading Behavior in Large Blog Graphs Patterns and a model [Internet]. https://cs. stanford.edu/people/jure/pubs/blogs-sdm07.pdf.

18. Lee K. The Biggest Social Media Science Study: What 4.8 Million Tweets Say About the Best Time to Tweet [Internet]. https://blog. bufferapp.com/best-time-to-tweet-research (cited 30 Jan 2018).

19. Twitter. Twitter Analytics. https://analytics.twitter.com/about.

20. Adams CE, Friedel JS, Jayaram M, et al. Which days to Tweet? A study protocol for a randomised controlled trial. ResearchGate 2017.

21. Taylor P. When is approval needed? 2011. http://www.orei.unimelb. edu.au/content/when-approval-needed (cited 1 May 2015).

22. NHS Health Research Authority, Medical Research Council. Do I need NHS REC approval? 2013. http://www.hra-decisiontools.org. uk/ethics/ (cited 1 May 2015).

23. Mental Elf [Internet]. http://www.nationalelfservice.net/mental-health/ (cited 16 Nov 2015)

24. SocialBro - The \#1 marketing platform for Twitter [Internet]. http:// www.socialbro.com/ (cited 30 Apr 2015)

25. Best Frequency Strategies: How Often to Post on Social Media [Internet]. https://blog.bufferapp.com/social-media-frequency-guide (cited 30 Oct 2017).

26. Inc HM. Social Media Marketing \& Management Dashboard [Internet]. https://hootsuite.com/ (cited 2017 Oct 30).

27. HootSuite launches in Chinese + Integrates Sina Weibo - Hootsuite Social Media Management [Internet]. https://blog.hootsuite.com/ chinese-localization-weibo/ (cited 30 Oct 2017).

28. Mason H. Time Is On Your Side [Internet]. 2012 http://blog.bitly.com/ post/22663850994/time-is-on-your-side (cited 2014 Jul 2).

29. Kupavskii A, Ostroumova L, Umnov A, et al. Prediction of retweet cascade size over time. Proceedings of the 21st ACM international conference on Information and knowledge management [Internet]: ACM Press, 2012.:2335-8.

30. Supermetrics [Internet]. Supermetrics. [https://supermetrics.com/ (cited 2017 Jul 5).

31. Adams CE, Jayaram M, Bodart AY, et al. Tweeting links to Cochrane Schizophrenia Group reviews: a randomised controlled trial. BMJ Open 2016:6:e010509.

32. Dissemination and 'impact' [Internet]. http://www.ethicsguidebook. ac.uk/Dissemination-and-impact-144 (cited 30 Jan 2018).

33. Carpenter D, Nieva V, Albaghal T, et al. Development of a Planning Tool to Guide Research Dissemination. Programs, Tools, and Products. Rockville, MD: Agency for Healthcare Research and Quality (US), 2005. 